\title{
Lyman $\alpha$ Observations as a Possible Means for the Detection of the Heliospheric Interface
}

\author{
Eric Quémerais, Rosine Lallement, and Jean-Loup Bertaux
}

Service d'Aéronomie du Centre National de la Recherche Scientifique, Verrières le Buisson, France

\begin{abstract}
We first summarize our current knowledge of the flow of interstellar $\mathrm{H}$ and He gas through the solar system. Both come from the same direction, but the $H$ velocity is $20 \mathrm{~km} / \mathrm{s}$, whereas the $\mathrm{He}$ velocity is $26 \mathrm{~km} / \mathrm{s}$ as recently determined by Ulysses, identical to the velocity found for the local interstellar cloud (LIC) as recently determined from absorption lines detected in the spectrum of nearby stars. This velocity difference may be assigned to the deceleration of $\mathrm{H}$ atoms at the heliospheric interface through coupling with the partially ionized interstellar plasma. Seen from the inner solar system, the Lyman $\alpha$ emission pattern of $H$ atoms (resonance scattering of solar photons) is quite compatible with a standard model including no interaction for $\mathrm{H}$ at the heliopause and therefore cannot be used to characterize such an interaction. We investigate three other types of Lyman $\alpha$ observations which could bear the signature of the heliopause. First, it is shown from Monte Carlo modeling of the interface perturbation that the Lyman $\alpha$ line profile (accessible through high resolution spectroscopy from Earth orbit) varies in a different way from upwind to downwind direction, whether there is a perturbation or not at the heliopause. Indeed, some Prognoz data show such a behaviour, and the potential of planned future observations is discussed (HST and SOHO). Second, since some interaction models predict a decrease of $\mathrm{H}$ density at heliopause crossing along the wind (or $\mathrm{H}$ increase when cruising upwind), the slope of the radial antisolar Lyman $\alpha$ intensity between 15 and $50 \mathrm{AU}$, characterized by $\gamma$, such as $I \approx k r^{\gamma}$, is computed for various models of $H$ density. It is shown that (1) the value of $\gamma$ recorded along downwind trajectories depends too much on solar parameters (radiation pressure and ionization) to be very useful to detect a departure from a standard model; (2) the value of $\gamma$ along upwind trajectories is much less sensitive to solar parameters but radiative transfer of Lyman $\alpha$ in the interplanetary medium must absolutely be taken into account for a correct interpretation; (3) if taken at face value, the value $\gamma=-0.78$ reported for Voyager data between 15 and 50 astronomical units cannot be explained with any standard model, and calls for an increase of density somewhere further upwind. A good fit is obtained with a doubling of the density at 54 astronomical units (hydrogen wall), but this is not a unique solution. Finally, the use of Lyman $\alpha$ intensity maps recorded at large distances $(30-50 \mathrm{AU})$ is considered. The shape of the map is different when a hydrogen wall is present, and this method is less prone to instrumental drift and solar Lyman $\alpha$ changes than the preceding one. The first method works whatever is the distance of the heliopause, whereas the two others require observations from upwind region, not too far from the heliopause (a few tens of astronomical units at most).
\end{abstract}

\section{INTRODUCTION}

The flow of $\mathrm{H}$ and He atoms through the solar system, called the interstellar wind, is a result of the relative motion of the Sun in respect to the surrounding interstellar material. Through resonance scattering of solar photons at $\mathrm{H}$ Lyman $\alpha(121.6 \mathrm{~nm})$ and He I $58.4 \mathrm{~nm}$, one can observe these atoms at a solar distance of $\approx 0.5$ to a few astronomical units from spacecraft in Earth's orbit or cruising the inner solar system. This "interstellar glow" was predicted by Blum and Fahr [1970] and measured for the first time in 1970 [Bertaux and Blamont, 1971; Thomas and Krassa, 1971]. $H$ atoms are strongly ionized by charge exchange with solar wind protons (the newly created neutral is so fast that it is no longer illuminated by the solar Lyman $\alpha$ line) and by EUV solar radiation $(\lambda \leq 91.2 \mathrm{~nm})$, which accounts for about $20 \%$ of the total ionization. The sky Lyman $\alpha$ pattern as observed from 1 astronomical unit is characterized by a smooth variation between a wide maximum region of intensity $\approx 600 \mathrm{R}$ and a minimum region in the opposite direction of about $200 \mathrm{R}$. The obvious interpretation is that the interstellar wind is coming from the region of maximum

Copyright 1993 by the American Geophysical Union.

Paper number 93JA01180.

0148-0227/93/93JA-01180 \$05.00 emission (the upwind direction); in the downwind direction, the ionization cavity carved by the sun is partially filled because of the high temperature of the interstellar gas.

The use of a Hydrogen absorption cell on Prognoz 5 and 6 allowed to analyze the interplanetary Lyman $\alpha$ spectral line width and position, and to derive the temperature $T_{\infty}=$ $8000 \pm 1000 \mathrm{~K}$ and the velocity $V_{\infty}=20 \mathrm{~km} / \mathrm{s}$ of the interplanetary $\mathrm{H}$ gas flow [Bertaux et al., 1985]. The upwind intensity was compatible with a density "at infinity" $n_{\infty}=$ $0.065 \mathrm{~cm}^{-3}$.

The direction of the incoming $\mathrm{H}$ flow was found to be identical to the axis of the helium cone, present downwind as a result of gravitational focusing: ecliptic longitude $\lambda=$ $254 \pm 3^{\circ}$, ecliptic latitude $\beta=7 \pm 3^{\circ}$, that is to say near the ecliptic plane and (by coincidence) near the direction of the galactic center.

Since the local interstellar matter is likely to be partially ionized, the plasma flow of the interstellar wind will interact with the solar wind plasma, with possibly a heliopause separating the two plasmas (Figure 1). The nose of the heliopause, or the nearest point of interstellar matter unperturbed by the heliosphere is to be found in the direction of the incoming interstellar wind. For further details on the modeling of the heliospheric interface, see, for example, Holzer [1989], Baranov et al. [1991] and Osterbart and Fahr [1992]. 


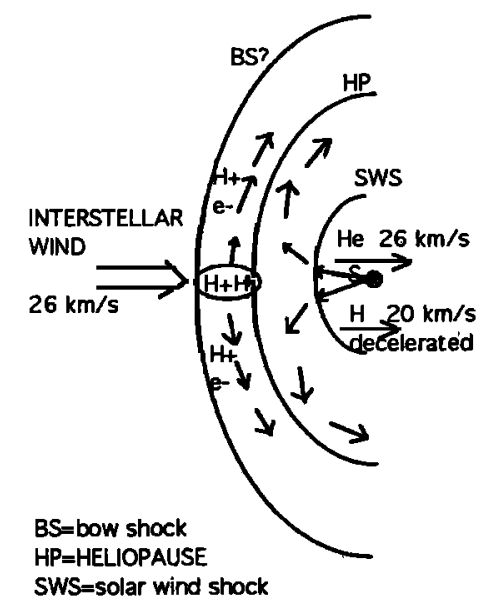

Fig. 1. Schematic view of the heliospheric interface. Neutral helium is unaffected while flowing through the diverted and decelerated plasma, as shown by the equality of velocity and temperature in the local cloud and within the heliosphere. Neutral H atoms distribution is likely to be affected by the plasma through charge exchange with $\mathrm{H}^{+}$, and decelerated.

As suggested by Wallis [1975], the interstellar plasma (mainly protons and electrons) may be decelerated and deviated by the heliospheric obstacle (Figure 1), and by $\mathrm{H}-\mathrm{H}^{+}$ resonance charge exchange, the $\mathbf{H}$ neutrals are somewhat coupled to the plasma. As a result, we do not know if the $\mathrm{H}$ parameters derived from Lyman $\alpha$ observation in the inner heliosphere $\left(n_{\infty}, T_{\infty}, \vec{V}_{\infty}\right)$ are the interstellar values out of the heliosphere, or if they represent only the gas inside the heliosphere, after modifications at the heliospheric interface. On the contrary, neutral helium is very unlikely to be affected by the plasma (Figure 1).

Several models of the neutral $\mathrm{H}$ interaction with the plasma at the interface have been presented and some of them predict a decrease of the density, a decrease of the velocity and some heating. The case of a subcritical interstellar flow has been considered by Fahr and Ripken [1984], Osterbart and Fahr [1992], whereas in the case of a supersonic two-shock model a Monte Carlo simulation of $\mathrm{H}$ atom trajectories was performed [Malama, 1991; Baranov et al., 1991].

Different ways to infer quantitative estimates of the neutral $\mathrm{H}$ perturbations (mainly the deceleration) have been considered, as the comparison between the neutral solar system $\mathrm{H}$ and He velocities. One way is the comparison between the "external" $\mathrm{H}$ velocity (the local interstellar gas velocity), and the "internal" solar sytem velocity [Lallement et al, 1986]. The local interstellar cloud (LIC) is the name given to the particular parcel of interstellar gas in which the sun is presently moving. Interstellar clouds can be detected from their absorption lines recorded in high-resolution spectra of various stars, but up to now it had been impossible to tell if one particular observed absorption was due to the LIC or was due to a cloud further away, between the Sun and the target star (or embedding the target star), because of the complexity of the local gas [Lallement et al., 1986, 1990]. But, finally, the whole subject became suddenly much less speculative since the recent use of northern hemisphere high-quality observations which have led to the detection of the LIC [Lallement and Bertin, 1992]. They analyzed a series of ground-based high-resolution spectra of nearby stars emcompassing a wide portion of the sky, obtained at
Observatoire de Haute Provence (France), and derived the velocity vector of $\vec{V}_{\text {LIC }}$ of the local cloud, strongly confirmed by one Hubble Space Telescope observation by Linsky et al. [1992]. The direction of $\vec{V}_{\mathrm{LIC}}$ motion of the LIC respective to the sun, $\left(\lambda=74.9 \pm 3^{\circ}, \beta=-7.8 \pm 3^{\circ}\right)$, is nearly the same as the direction of $\vec{V}_{\infty}$, adding a new evidence that the detected clond was the local interstellar cloud. For further details, see Bertin et al. [this issue].

However, the velocity was found to be $25.7 \pm 1 \mathrm{~km} / \mathrm{s}$, different from the $20 \pm 1 \mathrm{~km} / \mathrm{s}$ observed for $H$ in the solar system. But at the same time, Witte et al. [1992] reported their Ulysses measurements of the flow of He atoms near Jupiter, by direct detection of impact of He atoms on a litium fluoride target. See Witte et al. [1992] for a detailed description of this new technique. They found that the helium flow through the solar system is characterized by a velocity vector $\vec{V}_{\mathrm{He}}\left(\lambda=72 \pm 2.4^{\circ}, \beta=-2.5 \pm 2.7^{\circ}\right)$ and a modulus of $25.9 \mathrm{~km} / \mathrm{s}$, that is to say a vector with the same velocity modulus as $\vec{V}_{\text {LIC }}$ and nearly the same direction.

Therefore two things can now be concluded :

1. The helium flow in the solar system is identical to the motion of the LIC, proving that Helium atoms are not decelerated at the heliospheric interface.

2. If one refers to the most precise measurement of its velocity, the hydrogen flow is found to be decelerated by about $6 \mathrm{~km} / \mathrm{s}$, proving the existence of a strong interaction near the heliopause.

The mere fact that the $H$ flow inside the solar system is found at $20 \mathrm{~km} / \mathrm{s}$ instead of $26 \mathrm{~km} / \mathrm{s}$ for Helium gives an important clue to the effect of the heliospheric interface, and in a sense this was recognized a long time ago, when Dalaudier et al. [1984] had found, from optical measurements of He $58.4 \mathrm{~nm}$ emission, a helium velocity of $27 \pm 3$ $\mathrm{km} / \mathrm{s}$. However, they had also found a rather large temperature of He, not confirmed by Ulysses measurements, and Chassefière et al. [1988] had given another interpretation of the same measurements, in which the He velocity necessary to fit the optical data could be pushed down to $20 \mathrm{~km} / \mathrm{s}$, if the exciting solar line was itself strongly shifted. In fact, optical measurements of He do suffer from a lack of accurate knowledge of the solar line, and the recent Ulysses direct measurements put an end to this controversy.

In this paper, we examine how Lyman $\alpha$ measurements could bring more information on the distant region of interaction, near the heliopause. As seen from the inner solar system, the Lyman $\alpha$ intensity pattern can be perfectly represented by a standard "hot model," with no need to invoke anything special at the heliopause (with the inclusion of some variations of the solar wind ionization rate with latitude). But there are other types of measurements, either with a refined analysis of the spectral profile of the backscattered Lyman $\alpha$ line, or intensity measurements at large heliocentric distances, which may carry the imprint of the interaction near the heliopause.

In the first section we review recent studies on the spectral signature of the heliospheric perturbations in the hydrogen glow. In particular, Monte Carlo simulations of $\mathrm{H}$ atoms trajectories, when taking into account the interaction with the interstellar and solar plasmas before entering the heliosphere, and the resulting Lyman $\alpha$ line shapes predicted to be observed from 1 astronomical unit, show that these Lyman $\alpha$ line shapes have some peculiarities which do not appear in simple hot models without interface. Indeed, ac- 
curate line shape measurements could bear the signature of the heliospheric interface.

In the second section we analyze the behavior of antisolar Lyman $\alpha$ intensity measurements as a function of the heliocentric distance $r$, in both downwind and upwind directions. It is shown that downwind observations give a poor diagnostic, because the effect of ionization can change in a large range the radial dependence of the intensity, whereas upwind, an increase of density when going ontside seems to fit Voyager observations presented by Gangopadhyay and Judge [1992]. A full model including radiative transfer of Lyman $\alpha$ in the interplanetary space was build for this purpose.

In the third section we examine the possibility to detect an increase of density from a sky map obtained at a large distance

( 30 or 45 astronomical units) but still before entering the region of increased density. Here again, a full model including the radiative transfer was used, which is absolutely necessary to make correct predictions.

\section{Lyman $\alpha$ Line Profiles}

2.1. Line Profiles in the Case of an Homogeneous Flow: Sensitivity to Interstellar and Solar Parameters

At present (and within the next decade), UV spectrometers with a high enough resolution to measure the shape and the Doppler shift of the Lyman $\alpha$ lines are (or will be) orbiting the Earth or within 2 astronomical units, and then we will concentrate on the characteristics of the lines as viewed from 1 astronomical unit. So close to the Sun, hydrogen atoms are significantly affected by solar gravitation, radiation pressure, and ionization, and the determination of the interstellar velocity $V_{\infty}$ and temperature $T_{\infty}$ far from the sun, (maybe already affected by the interface), suffers from this complication, Within a few astronomical units, the bulk velocity is spatially dependent, and, due to the solar cycle radiation and solar wind changes, it also varies with time. Resulting line profiles variations have been studied in the case of homogeneous flows approaching the Sun and optically thin radiation field [ $W u$ and Judge, 1980; Lallement et al., 1985]. The main solar effect remains the one of the $\mu$ parameter (ratio of Lyman $\alpha$ radiation pressure to gravitation). In the case of high, respectively low, solar Lyman $\alpha$ flux, $\mathrm{H}$ atoms are decelerated, respectively accelerated, when approaching the Sun and then return to their initial velocity when moving away on the downwind side. It is interesting to note that, even in the simple case where $\mu$ is equal to 1 i.e., radiation pressure balances gravitation, if all trajectories are straight lines with constant velocity for each atom, the bulk velocity is significantly changed both in modulus and direction due to selection effects during the ionization processes (fast atoms, spending less time near the sun, are less ionized).

Fortunately, when using profiles measured toward different directions, it is possible to disentangle the profile characteristics related to $\mu$ from those related to the interstellar parameters $\vec{V}$ (velocity) and $T$ (temperature) far from the Sun and to measure these parameters independently, as was done with the already mentioned hydrogen cell experiments.

This classical modeling of the Lyman $\alpha$ emission profile however, failed to explain the angular evolution of the emission profile when turning the direction of sight towards the downwind side as observed with Prognoz $\mathrm{H}$ absorption cell in 1976-1977 [Bertaux et al., 1985]. For more detajls see
Lallement [1990]. The discrepancies between the upwind and downwind observations suggest that the red part of the downwind line (reflecting $\mathrm{H}$ atoms leaving the heliospheric cavity with the highest velocity) is enhanced with respect to what is expected from upwind profiles. This discrepancy has been attributed to different types of physical processes. The most probable reasons are; (1) departures from an homogeneous flow due to the heliospheric interface perturbations; (2) multiple scattering of Lyman $\alpha$ photons; (3) time-dependent effects.

While in any case full radiative transfer line profile calculations and time-dependence inclusion are certainly necessary improvements of the models (indeed, radiative transfer codes have already been proven to change significantly at least the intensity field, as shown in section 3 , the first explanation appears as the most appropriate, as we will show below.

\subsection{Quantitative Estimates of Lyman $\alpha$ Line Profiles Variations Induced by Heliospheric Interface}

One could imagine at first sight that the hydrogen flow having crossed the heliospheric interface is characterized by a new distribution function, by comparison with the unperturbed interstellar conditions, and that the first orders moments $n, V, T$ of this distribution would be the same for the rather small volume of gas illuminated by the Sun and contributing to the glow. In this case, one should be able to determine these parameters $n, V, T$ with the help of a classical homogeneous flow model without interface; simply the derived values would apply to the postinterface gas (and not to the initial interstellar flow), and we would have no sign at all of the initial conditions by looking at hydrogen only.

This is not true, for the very simple reason that the gas which is detected along the stagnation line on the upwind side and the gas which is detected on the downwind axis do not come from the same direction: they have crossed the interface in different regions characterized by different plasma properties, and then have started far from the Sun with different distribution functions (Figure 1). In other words, while they are at the same time rather close to the Sun in a small region, their history is different. This is due to the "umbrella effect" of the sun: schematically, $H$ atoms able to reach the middle of the downwind cavity can not come directly from the stagnation axis, since they would have been ionized by the Sun, they come from the "sides," and they have crossed the flanks of the interface, where the plasma is less decelerated and heated. It results that, depending on the direction the Lyman $\alpha$ emission comes from, one probes different initial properties of the emitting neutrals, i.e., different distribution characteristics the scattering neutrals had before approaching the sun [Lallement and Bertaux, 1990]. This gives a natural explanation to the upwind to downwind profiles discrepancies discussed above, provided the hydrogen distribution is significantly affected by the interface.

To quantify this effect, which has the interesting property of being independent of the distance to the heliopause and the shock(s), two different approaches have been used. The first way is to start with an empirical inhomogeneous $H$ distribution far from the Sun, for which the velocity and temperature vary with the distance to the stagnation line, to model all the flow tubes according to this distribution, and vary the initial distribution of $\vec{V}_{\infty}$ and $T_{\infty}$ to fit the data. 
The second way relies on the computation of the upwind and downwind line profiles for a full modelling of plasma interface and neutral $\mathrm{H}$ flow through the interface. This second approach requires an already fully developed plasma model. We will now describe these two approaches.

As we said, the $\mathrm{H}$ cell downwind observations of Prognoz suggest that the red part of the downwind line is enhanced with respect to what is expected from upwind profiles. The existence of faster atoms is in agreement with the hypothesis that $H$ is decelerated mostly along the stagnation line, while atoms seen downwind have flown further away from this line and are less decelerated. Quémerais et al. [1992] have empirically modeled an axisymmetric hydrogen distribution whith the following characteristics: far from the Sun, the initial bulk velocity and temperature is a function of the distance to the stagnation line, as would result from the coupling with an axisymmetric plasma interface. Then all atoms are followed during their travel through the heliospheric cavity, where they interact with the supersonic solar wind only. Resulting line profiles are computed, as well as the degree of absorption by a hydrogen cell, and the results are compared with observed Prognoz absorption rates. A systematic search for the velocity and temperature dependences on the distance to the stagnation lines which remove the best the previous homogeneous model discrepancies, is then performed.

The results are interesting in the following way: they show that, in order to fit the data, one has to increase the temperature and decrease the bulk velocity along the stagnation line, and inversely to decrease $T_{\infty}$ and increase $V_{\infty}$ on the "sides". This corresponds qualitatively with the expected modifications due to the physical processes at the interface. However, quantitatively, the required velocity and temperature modifications, are probably too large to be taken as real local values. As an example, the velocity decrease is of $\mathbf{1 6}$ $\mathrm{km} / \mathrm{s}$ in the example shown in Figure 2, where the expected and observed absorption rates by the $\mathrm{H}$ cell are displayed along a scan plane perpendicular to the ecliptic and nearly parallel to the flow. Also, there is a range of directionsof-sight, not shown here, for which significant discrepancies remain. Then probably this kind of modeling is quantitatively too crude and some other effects are present.

The second approach is the use of the Baranov interface model [Malama, 1991; Baranov et al., 1991], coupled with a Monte-Carlo simulation of the neutrals flow through the interface [Malama, 1991], to derive the Lyman $\alpha$ line profiles as observed from the inner solar system. The main idea was to quantify the upwind-downwind line profiles evolutional pattern and to compare this angular evolutional pattern with the no-interface case [Lallement et al., 1992]. As a matter of fact, this is an observable quantity. In Figure 3 is shown the upwind emission line profile resulting from the BaranovMalama model. For more clarity we show a case where the interstellar proton density has the rather high value of 0.2 $\mathrm{cm}^{-3}$. A classical homogeneous model profile is also shown, for which interstellar parameters have been adjusted to produce exactly the same profile as in the presence of the interface. The two corresponding downwind profiles, at $180^{\circ}$ from the previous direction, are shown in the same Figure. The profile which results from the full interface modelling is very different from the "classical profile" with no interface. In this particular case used for demonstration the neutral $\mathrm{H}$ is decelerated by $13 \mathrm{~km} / \mathrm{s}$ (from 29 to $16 \mathrm{~km} / \mathrm{s}$ ) along the stagnation axis, while heated from $7600 \mathrm{~K}$ up to $12000 \mathrm{~K}$. Along the sides, the velocity and temperature are less modified, being $21 \mathrm{~km} / \mathrm{s}$, and $9000 \mathrm{~K}$ respectively. Of course, the variations decrease with the interstellar plasma density, but it is interesting to note that there is still a detecTable difference for $0.02 \mathrm{~cm}^{-3}$, which is a reasonable value. This proves that the umbrella effect is really significant, namely that even if the emitting $H$ is confined within a rather small volume close to the sun, it comes from different portions of

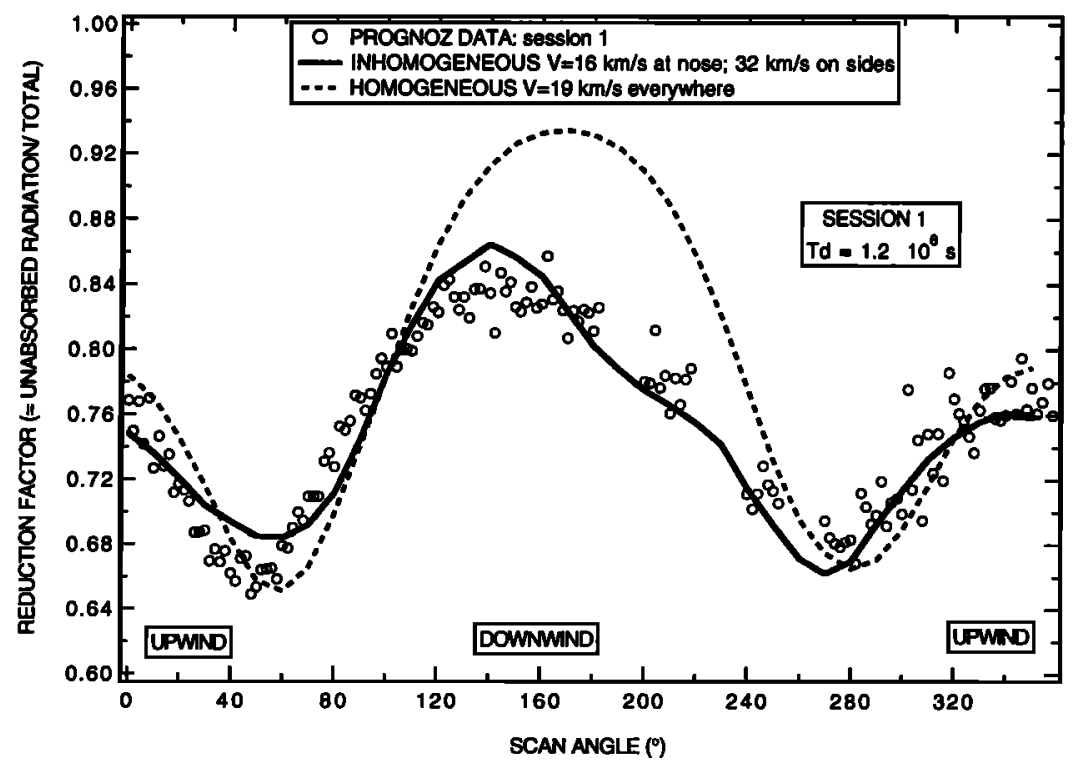

Fig. 2. This figure is adapted from Quémerais et al. [1992]. Absorptions by the prognoz $H$ cell are best fitted by an inhomogeneous hydrogen flow entering the heliospliere, with slower and warmer atoms along the stagnation line, as compared with a classical homogeneous How far from the Sun. The data of session 1 were obtained for an Earth ecliptic longitude of $11^{\circ}$ in a scan plane perpendicular to the Earth-Sun axis. The origin of the scan angle is defined by the intersection of the scan plane with the ecliptic plane, in the opposite direction of Earth motion. 


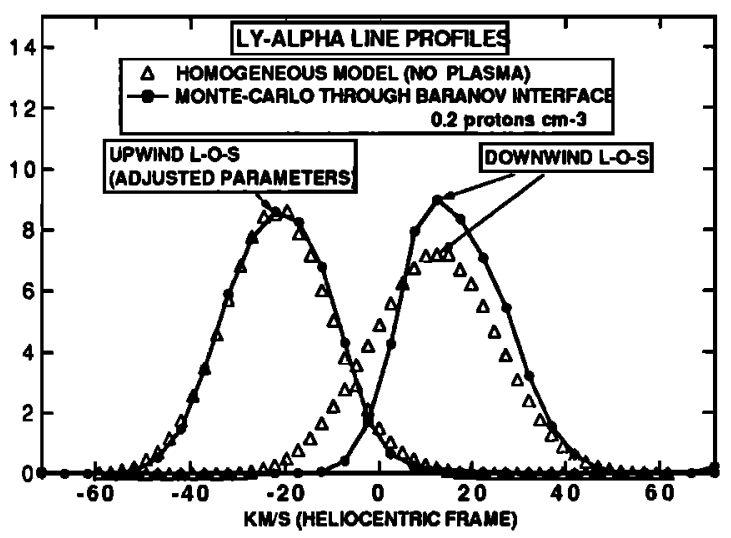

Fig. 3. This figure is adapted from Lallement et al. [1992]. The Lyman $\alpha$ line profile varies in a different way from upwind to downwind direction, wether there is a a perturbation or not at the heliospheric interface. Parameters have been adjusted to provide the same upwind profile.

the heliospheric interface. Therefore, by observing H Lyman $\alpha$ profiles, and their variations with direction of sight, one can learn something about the heliospheric interface.

\subsection{Past and Future Observations}

2.3.1. Spectroscopy. Spectra of the Lyman $\alpha$ emission lines have already been observed directly by Adams and Frisch [1976] with Copernicus (OAO-3), at a resolution of about $15 \mathrm{~km} / \mathrm{s}$, resulting in a bulk velocity determination of $22 \pm 3 \mathrm{~km} / \mathrm{s}$, and later by Clarke et al. [1984] with IUE at a resolution of $40 \mathrm{~km} / \mathrm{s}$, giving $V_{\infty}=25.5 \pm 5 \mathrm{~km} / \mathrm{s}$. The line width being of the order of $15 \mathrm{~km} / \mathrm{s}$ (full width at half maximum), it is clear that the error bars are due to the unfortunately too poor resolving power of the two instruments. The common interval $20.5-25 \mathrm{~km} / \mathrm{s}$ for these two measurements is marginally compatible with the hydrogen cell results $19-21 \mathrm{~km} / \mathrm{s}$ on the upwind side. There were strong hopes associated with the Hubble Space Telescope Goddard high resolution spectrograph (GHRS), which has a resolving power of $3 \mathrm{~km} / \mathrm{s}$ in the Echelle Mode, and a proposal has been accepted on this subject. The excellence of the instrument and the feasiblity of such an accurate measurement is largely proven by an observation obtained as a by-product of early Mars observations (J.L. Bertaux, private communication, 1992). After $20 \mathrm{~min}$ of integration, the interplanetary line is very well seen, showing that exposures of only 1 or two hours would have given very good results. Unfortunately, the low-wavelength part of $G H R S$ is no longer operating at this high resolution, delaying further measurements by an unknown number of years.

2.3.2. Hydrogen cell. The use of a hydrogen cell acting as a kind of negative spectrometer, limited to the spectral range implied by the spacecraft (or Earth's) motion with respect to the gas, but with infinite resolution, has been proven to bring very strong constraints on the line profiles. Indeed, the Prognoz $\mathrm{H}$ cell results are still a matter for analysis, as has already been discussed above.

The future $\mathrm{H}$ cell experiment SWAN which should fly on the solar and heliospheric observatory (SOHO) in 1995 for 2 years, has extended capabilities with respect to Prognoz, providing a full sky coverage at any time (instead of a fixed plan), and a better accuracy. It should provide extremely strong constraints on the shapes and shifts of the lines, allowing to measure the bulk velocities and to detect the departures from an homogeneous flow, when the line of sight moves from upwind to downwind. These departures, bearing the signature of the heliospheric interaction, could help describe the interface, whatever is its actual distance from the sun.

\section{RADIAL DEPENDENCE of ANTISOLAR INTENSITIES}

The outer-heliospheric spacecraft Voyager 1 and 2 and Pioneer 10 and 11 provide us with new means to study the Lyman $\alpha$ emissivity field in the heliosphere and then to get better constraints on the neutral hydrogen distribution in the heliosphere. Measurements of Lyman $\alpha$ intensities within the inner heliosphere (e.g., Prognoz 5/6, Pioneer-Venus...) are more influenced by the solar environment and its timedependent or latitudinal variabilities, whereas such effects are smoothed in the outer heliosphere.

Some studies from within the inner heliosphere have provided a better understanding of the flow of interstellar hydrogen [Lallement et al., 1985; Chassefière et al., 1986]. For instance, the interstellar hydrogen number density has been constrained between 0.05 and $0.2 \mathrm{~cm}^{-3}$. There are several reasons for this rather large uncertainty. The illuminating Lyman $\alpha$ solar flux at line center varies; the same measured intensity may correspond to different couples density and ionization rate. Calibration factors evaluation may also prove to be a difficult task and cause discrepancies between the results of different experiments. One way to rule out this kind of problem is to study radial dependence of intensities normalized to a chosen value.

On the other hand, as shown by various authors [Baranov et al., 1991; Fahr, 1990], the hydrogen density distribution in the outer heliosphere may consistently differ from what is expected by a classical hot model, as developped for instance by Thomas [1978] or Lallement et al. [1985] described by a uniform density at large distance from the Sun and a ionization cavity carved into it near the sun. Indeed, in the case of an interface between interstellar and solar plasmas, and since neutrals and protons are coupled through charge exchange processes, the hydrogen distribution in the outer heliosphere may differ greatly from the smooth distribution obtained in the absence of interface. Baranov et al. [1992] give some possible $\mathrm{H}$ distributions according to computations made with a Monte Carlo type model coupled with a plasma calculation. The main expected feature appears is the upwind direction. It consists of an important depletion of neutral hydrogen along the stagnation line, near the position of the heliopause and the stagnation point of the plasma distribution. The exact position of this feature is not well constrained yet, neither is its exact shape. In fact, this sharp depletion feature may also be coupled with a slow radial gradient of density between the Sun and the solar wind shock.

Then, radial variations of backscattered Lyman $\alpha$ intensities provide us with a means to study this kind of problem. Since some of the spacecraft are now as far as $\mathbf{5 0}$ astronomical units from the sun, it is shown below that this problem must not be studied by using the optically thin approximation and in what follows we have included radiative transfer as established by Quémerais and Bertaux [1992]. 


\subsection{Density Distribution Model With no Interface}

Our aim here is to study the radial variation of antisolar intensities when no interface is considered. Up to now such studies included the optically thin approximation which yields wrong conclusions for this problem.

In Figure 4 and 5 , we show the expected $H$ density distribution for some given solar parameters. The main feature is a depletion around the Sun elongated in the downwind direction thus leading to quite different behaviours in the upwind and downwind directions. The downwind part of the cavity will be more or less depleted according to the value of the solar parameters. A greater lifetime of one $H$ atom at 1 astronomical unit against ionization ( $T_{d}$ large), as well as a stronger focusing effect ( $\mu$ smaller than 1 ) will tend to fill this cavity. Such distributions derived from the classical hot model are relevant in the near-sun environment (say within 20 astronomical units from the sun) where effects of an interface can be neglected. These distributions are normalized to the density "at infinity" $n_{\infty}$ which is one of the parameters of the model. It must be noted that if this parameter represents the density number in LIC for a no-interface model, in the case of an interface model it represents the number density after filtration by the interface. Then, if the global effect of the interface is to divide the number density by a factor $q$ along the stagnation line, the number density in LIC is given by $n_{\ell c c}=q n_{\infty}$.

3.1.1. Optically thin approximation. Let $I(\vec{r})$ be the antisolar intensity measured at vector position $\vec{r}$. This quantity can be computed according to the optically thin approximation by

$$
I(\vec{r})=\frac{1}{4 \pi} \int_{r}^{\infty} \varepsilon_{o t}\left(\overrightarrow{r^{\prime}}\right) p(\pi) d r^{\prime}
$$

where $p(\omega)$ is the nonisotropic Lyman $\alpha$ phase function given by Brandt and Chamberlain [1959] and $\varepsilon_{o t}\left(\vec{r}^{\prime}\right)$ is the optically thin emissivity at $\overrightarrow{r^{\prime}}$. This value is proportional to the $H$ density at $\overrightarrow{r^{\prime}}$ and to the solar Lyman $\alpha$ flux. Then $\varepsilon_{o t}(\vec{r})$ is proportional to $n(\vec{r}) / r^{2}$, from which we see that, if $\partial n / \partial r \approx 0, I(\vec{r})$ must be proportional to $1 / r$.

It is obvious from Figure 4 that the condition $\partial n / \partial r \approx 0$ will be fulfilled only far away from the Sun (say 15 astronomical units in the upwind direction and 50 astronomical units downwind). Moreover since the density is an increasing function for increasing values of the distance from the sun, at least for no-interface models, then the antisolar intensity must fall off more slowly than $1 / r$ which is typical of constant density. As we will see later this result is not true when multiple scattering is taken into account.

We must now take into account the fact that for a realistic density distribution the density gradient is not zero and depends on the interval on which it is computed. In what follows the radial dependence of the intensity $I(\vec{r})$ is characterized by a coefficient $\gamma$ computed as the slope of a linear function $\log (I)=\gamma \log (r)+k$. But as mentioned before, the interval on which this approximation $I \approx k r^{\gamma}$ is made will change the result. To avoid effects of the nearsolar environment, we have chosen distances greater than 15 astronomical units. Yet, in the downwind direction at 15 astronomical units from the Sun the effects of the cavity are still rather important (Figure 4).

As an example, Table 1 displays the radial-dependence coefficient for three different intervals $([15,50],[50,100]$ and

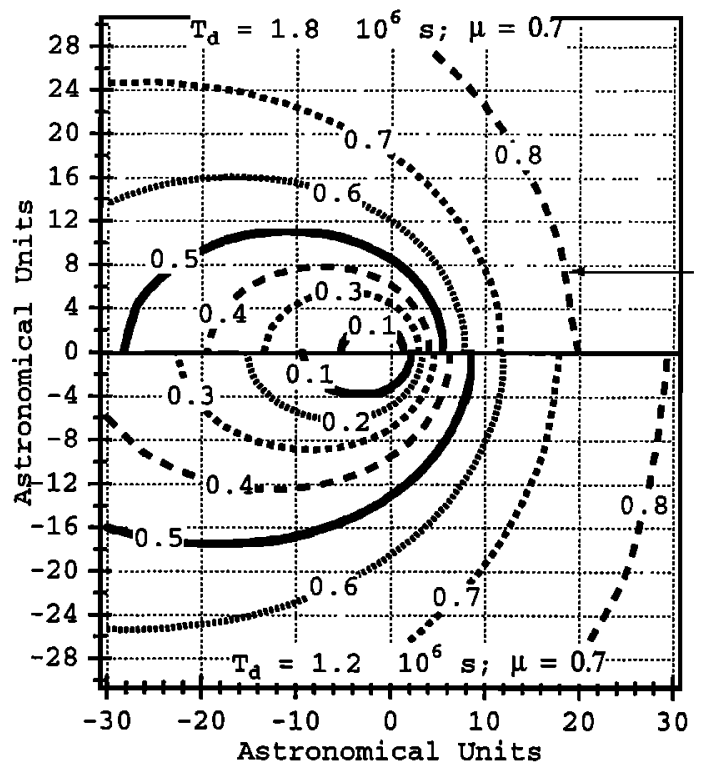

Fig. 4. Isodensity contours as expected from a classical hot model computed for two different sets of solar parameters. Upper graph: $T_{d}=1.8 \times 10^{6} \mathrm{~s}$ and $\mu=0.7$; lower graph: $T_{d}=1.2 \times 10^{6}$ $s$ and $\mu=0.7$. The density is normalized to its value at large distance from the sun. The direction of the incoming wind is shown by an arrow. The cavity is partly filled when $T_{d}$ increases (less ionization by solar fluxes). This effect is more effective in the downwind direction.

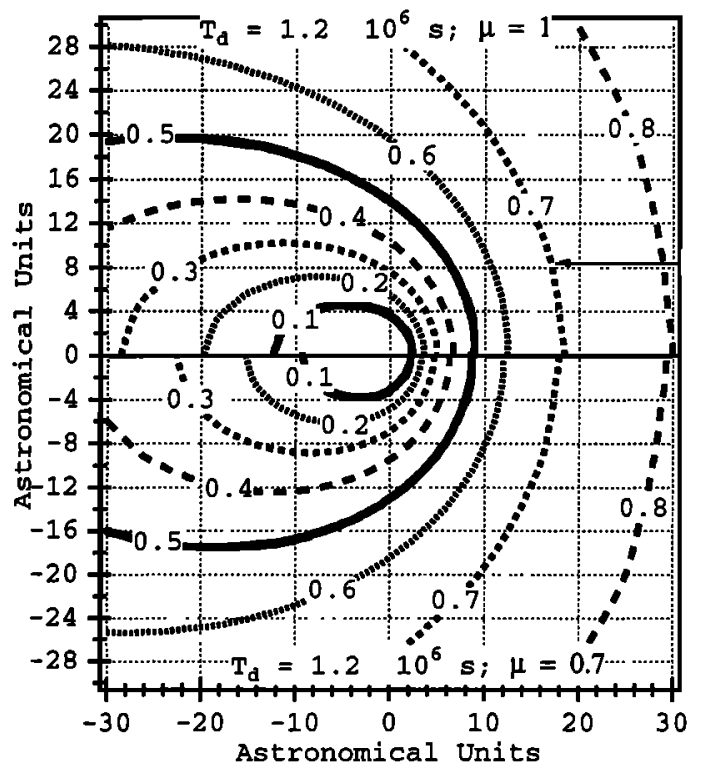

Fig. 5. Isodensity contours as expected from a classical hot model computed for two different sets of solar parameters. The density is normalized to its value at large distance from the sun. Upper graph: $T_{d}=1.2 \times 10^{6} \mathrm{~s}$ and $\mu=1$; lower graph: $T_{d}=1.2 \times 10^{6} \mathrm{~s}$ and $\mu=0.7$. When $\mu$ increases, focalisation of neutral atoms is less effective and the cavity is larger.

$[15,100])$ and three density models in the upwind and downwind directions. All models were computed for the same parameters of the interstellar wind $(20 \mathrm{~km} / \mathrm{s}$ and $8000 \mathrm{~K})$. The corresponding solar parameters are shown in Table 1 . We see from this Table that as the interval gets away from the Sun the coefficient tends toward the limit of -1 , which means that the density profile becomes flat as expected. This is 
TABLE 1. Radial Dependence Coefficient Computed for Three Different Models in an Optically Thin Approximation

\begin{tabular}{|c|c|c|c|c|c|}
\hline$\mu$ & $T_{d}$ & $\theta$ & $\stackrel{\gamma}{\gamma-50}$ & $\stackrel{\gamma}{50-100}$ & $\begin{array}{c}\gamma \\
15-100\end{array}$ \\
\hline 1 & $1.210^{6} \mathrm{~s}$ & $0^{\circ}$ & -0.88 & -0.95 & -0.90 \\
\hline 0.7 & $1.210^{6} \mathrm{~s}$ & $0^{\circ}$ & -0.88 & -0.96 & -0.90 \\
\hline 1 & $1.210^{6} \mathrm{~s}$ & $180^{\circ}$ & -0.56 & -0.77 & -0.66 \\
\hline 0.7 & $1.210^{6} \mathrm{~s}$ & $180^{\circ}$ & -0.60 & -0.79 & -0.67 \\
\hline 0.7 & $1.810^{6} \mathrm{~s}$ & $180^{\circ}$ & -0.72 & -0.86 & -0.78 \\
\hline
\end{tabular}

Outside 15 astronomical units, $\gamma$ does not depend on the model in the upwind direction, whereas it is model-dependent in the downwind direction, according to the size of the downwind cavity.

quite true for the upwind direction which consequently show very little model dependence whereas the cavity effect is still rather important in the downwind direction even when the lifetime against ionization of $\mathrm{a} \mathbf{H}$ atom at 1 astronomical unit is taken rather large $\left(1.8 \times 10^{6} \mathrm{~s}\right)$ which tends to fill the downwind cavity.

It must be pointed out that if the radial dependence of intensity beyond 15 astronomical units observed by Pioneer 10 in the downwind direction is equal to -1.07 [Gangopadhyay and Judge, 1992], the use of the optically thin approximation leads us to the conclusion that the classical hot model is inadequate to describe the downwind neutral distribution beyond 15 astronomical units. Such a value suggests that the density in the downwind direction is fairly constant (even decreases a little with increasing distance from the Sun) which cannot be obtained from hot models with realistic parameter values.

3.1.2. Multiple scattering model. For a temperature of $8000 \mathrm{~K}$ and far from the Sun, the $\mathrm{H}$ optical thickness per astronomical unit is of the order of the density "at infinity" $n_{\infty}$ expressed in $\mathrm{cm}^{-3}: \tau(1 A U) \approx n_{\infty}\left(\right.$ in $\left.\mathrm{cm}^{-3}\right)$, we see that the present positions of the outer heliospheric spacecraft do not justify the use of an optically thin approximation (O.T.).

A more complete modeling of radiative transfer for a resonance line must take two more effects into account. First, a photon can be scattered many times and then contribute more than once to the emissivity field. Then, that there is extinction along the path of the photon which depends on its wavelength. The first effect tends to increase the real intensity respective to the $O$.T. approximation, whereas the second tends to decrease it; but these two effects do not compensate each other exactly.

In what follows, we have used a model developed by $Q u e ́-$ merais and Bertaux [1992], which combines a Monte Carlo type code with a numerical code based on considerations similar to the work on the geocorona developped by Thomas [1963] or Anderson and Hord [1977]. The main simplifications used in this work are complete frequency redistribution (CFR) which, according to Thomas [1963] tends to slightly overestimate the emissivity field, along with an isotropic emissivity function. The phase function expression given by Brandt and Chamberlain [1959] shows that the maximum discrepancy is of about $15 \%$, yet since here we are only interested in gradients and not in absolute values the overall effect must be less important.

According to these assumptions, the antisolar intensity can now be expressed as

$$
I(\vec{r})=\int_{-\infty}^{+\infty} d \nu \frac{a(\nu)}{4 \pi} \int_{r}^{\infty} \varepsilon\left(\overrightarrow{r^{\prime}}\right) e^{-\tau_{\nu}\left(\vec{r}^{\prime}, \vec{r}^{\prime}\right)} d r^{\prime}
$$

where $a(\nu)$ expresses the spectral dependence of the absorption and scattering profiles and $e^{-\tau_{\nu}}$ is the extinction along the line of sight. The emissivity field $\varepsilon(\vec{r})$ takes multiple scattering into account.

For Doppler profiles at the temperature of the gas $\left(T_{\infty}=\right.$ $8000 \mathrm{~K}$ ), this expression becomes

$$
I(\vec{r})=\frac{1}{4 \pi} \int_{r}^{\infty} \epsilon\left(\vec{r}^{\prime}\right) T\left(\tau\left(\overrightarrow{r^{\prime}}, \vec{r}\right)\right) d r^{\prime}
$$

where $\tau\left(\overrightarrow{r^{\prime}}, \vec{r}\right)$ is the optical thickness at line center between $\overrightarrow{r^{\prime}}$ and $\vec{r}$ and $T(\tau)$ is the Holstein function given by

$$
T(\tau)=\frac{1}{\sqrt{\pi}} \int_{-\infty}^{+\infty} e^{-x^{2}} e^{-\tau e^{-x^{2}}} d x
$$

In this case the intensity gradient will depend on the behaviour of the extinction function represented by $T(\tau)$ and of the emissivity function $\varepsilon(r)$. Of course, close to the Sun the behaviour is similar to that of the optically thin approximation, yet for larger values of $\tau$ the gradient becomes consistently different from what is expected from optically thin computations. In fact, extinction tends to become prominent over the multiple scattering effect which results in the fact that for values of $n_{\infty}$ between 0.05 and $0.2 \mathrm{~cm}^{-3}$ the gradient is always steeper than what is expected from optically thin calculations : the denser the medium, the steeper the radial gradient of antisolar intensity.

The results of different computations have been summarized in Table 2 . First, we see that the radial gradient can be steeper than -1 which is coherent with the fact that the optically thin approximation overestimates the emissivity of far away points by neglecting extinction. We find also that for no-interface models the upwind gradient is always steeper than the downwind gradient over the same intervals, simply because of the existence of the downwind cavity.

Gangopadhyay and Judge [1992] have recently reported that the Pioneer 10 data between 15 and 50 astronomical units, exploring the downwind cavity, can be represented with a slope $\gamma=-1.07$. They have claimed that this value, which is incompatible with any value obtained within the

TABLE 2. Radial Dependence Coefficient Computed for Different Models With Radiative Transfer Calculations

\begin{tabular}{ccccccc}
\hline$\mu$ & $t_{d}$ & $n_{\infty}$ & $\theta$ & $\begin{array}{c}\gamma \\
15-50\end{array}$ & $\begin{array}{c}\gamma-100 \\
\gamma\end{array}$ & $\begin{array}{c}\gamma-100 \\
1\end{array}$ \\
& 1.2 & 0.1 & $0^{\circ}$ & -1.01 & -1.36 & -1.10 \\
0.7 & 1.2 & 0.1 & $0^{\circ}$ & -1.03 & -1.38 & -1.11 \\
1 & 1.2 & 0.1 & $180^{\circ}$ & -0.68 & -1.20 & -0.90 \\
0.7 & 1.2 & 0.1 & $180^{\circ}$ & -0.71 & -1.21 & -0.91 \\
0.7 & 1.8 & 0.05 & $180^{\circ}$ & -0.76 & -1.13 & -0.90 \\
0.7 & 1.8 & 0.1 & $180^{\circ}$ & -0.85 & -1.28 & -1.01 \\
0.7 & 1.8 & 0.15 & $180^{\circ}$ & -0.96 & -1.36 & -1.12 \\
0.7 & 1.8 & 0.2 & $180^{\circ}$ & -1.03 & -1.40 & -1.18 \\
\hline
\end{tabular}

All the models were computed for interstellar parameters 8000 $\mathrm{K}$ and $20 \mathrm{~km} / \mathrm{s}$. The solar parameters are indicated in the Table, where $t_{d}$ is the lifetime at 1 astronomical unit divided by one million seconds. $\theta$ is the angle of the direction with upwind. The intervals used to compute $\gamma$ are indicated. $\theta=0^{\circ}$ corresponds to the upwind direction and $180^{\circ}$ to the downwind direction. 
frame of the O.T. approximation and a standard hot model (Table 1), was an evidence that the real downwind $\mathrm{H}$ density distribution was different from the standard model and called for a perturbation caused at the heliospheric boundary. It is interesting to note that it is however possible to obtain a coefficient $\gamma$ equal to the value given by the Pioneer 10 data with the standard model though, in the framework of radiative transfer model (last line of Table 2), a rather large number density $n_{\infty}$ is required $\left(0.15-0.2 \mathrm{~cm}^{-3}\right)$ as well as a large value for the lifetime at 1 astronomical unit $\left(\approx 2 \times 10^{6} \mathrm{~s}\right)$ in order to decrease the size of the downwind cavity. Taken alone, the downwind intensity gradient is probably not a very good indicator of the effect of the interface on the $\mathrm{H}$ density distribution.

\subsection{Density Distribution Model With Wall Type Interface}

In the previous paragraph we have shown that, beyond 15 astronomical units in the upwind direction, the radial dependence coefficient $\gamma$ was not very dependent on the solar parameters. Radiative transfer models give a value close to -1 (see Table 2) on the interval 15-50 astronomical units.

It is then interesting to compare such results with the Voyager 1 and Voyager 2 Lyman $\alpha$ data. These data yield a value of -0.7 for Voyager 1 and -0.78 for Voyager 2 [Hall et al., 1993; Gangopadhyay and Judge, 1992]. This seems to be in contradiction with a hot model with no interface and these gradient values may be the signature of some jump of density lying ahead of the Voyager spacecraft which cruise outwards near the upwind direction.

Since the imprint of a shock on the $\mathrm{H}$ neutrals is not well constrained yet, we have simply assumed that, at a given position in the upwind direction, the screening effect due to the interface could be represented by a jump in density. Then, choosing one of the models of Figure $4(\mu=0.99)$, the number density factor $n_{\infty}$ was taken equal to $0.2 \mathrm{~cm}^{-3}$ for points with values of $r>R_{s}$ and with an angle with the upwind direction less than $40^{\circ}$ and equal to $0.1 \mathrm{~cm}^{-3}$ for all other points. The density distribution is shown in Figure 6. At the position of the shock, the density is multiplied by 2 which is coherent with the results of Baranov et al. [1991]. We tried three different values for $R_{s}, 54$ astronomical units, 80 astronomical units and 107 astronomical units, to estimate find which position better agreed with the Voyager data.

In Table 3, we display the results of our computations for these density distributions. If the shock is at $\mathbf{5 4}$ astronomical units, the slope $\gamma=-\mathbf{0 . 7 8}$ is quite different from the no-shock case at $\gamma=-1.01$. The value of $n_{\infty}$ inside the shock is equal to $0.1 \mathrm{~cm}^{-3}$, which means that an optical thickness of 1 roughly corresponds to 10 astronomical units. Therefore, from a vantage point at 50 astronomical units, only 4 astronomical units (or $\tau \approx 0.4$ ) away from the $\mathrm{H}$ wall, it is not surprising that the intensity is very much influenced by the presence of this wall. However, even if the $H$ wall is at 80 astronomical units, $\gamma=-0.89$ is significantly different from -1.01 , in spite of the optical thickness of 3 which separates the last observing position at 50 astronomical units and the $H$ wall.

On the other hand, for radiative transfer computations, the no-shock model (which is equivalent to $R_{s}=\infty$ ) and the model with $R_{s}=107$ astronomical units show the same gradient between 15 and 50 astronomical units. These two density distributions cannot be distinguished. The shock at 80 astronomical units modifies the gradient mainly for the

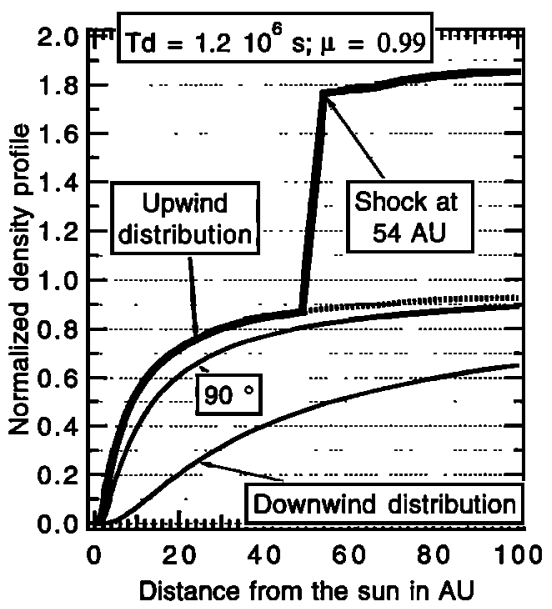

Fig. 6. Radial density distribution plotted for three different values of $\theta$, the angle with the upwind direction, in the case of a $\mathrm{H}$ wall. For $\theta$ greater than 40 deg or $r$ smaller then $R_{s}$ (here $R_{s}=54$ astronomical units) we have a classical hot model with $\mu=0.99$, $T_{d}=1.2 \times 10^{6} \mathrm{~s}$ and $n_{\infty}=1$ (normalization), for $\theta$ less than $40^{\circ}$ and $r$ greater than $R_{s}$ we take $n_{\infty}=2$.

TABLE 3. Upwind Radial Dependence Coefficient With Shocks

\begin{tabular}{ccc}
\hline$R_{\text {g in AU }}$ & $\begin{array}{c}\gamma(15-50) \\
\text { Optically Thin }\end{array}$ & $\begin{array}{c}\gamma(15-50) \\
\text { Radiative Transfer }\end{array}$ \\
\hline no shock & -0.88 & -1.01 \\
107 & -0.67 & -0.99 \\
80 & -0.62 & -0.89 \\
54 & -0.56 & -0.78 \\
\hline
\end{tabular}

Upwind radial dependence coefficient computed for four different density models, three of them with a shock at position $R_{s}$ in the upwind direction. Optically thin and radiative transfer results are compared.

larger distances in the interval [15 $\mathrm{AU}, 50 \mathrm{AU}]$. We must realize also that, due to the important increase of the primary emissivity in the shock region as compared to the nointerface case, points closer to the Sun than the shock have an increased secondary emissivity, due to photons backscattered from the shock region. Then the secondary emissivity of the preshock region is increased as compared to the expected secondary emissivity in absence of the shock even if locally the density distributions are the same. This higher secondary emissivity becomes a telltale sign of the shock, which may be itself too remote to be seen. We think this effect explains the fact that the gradient between 15 and 50 astronomical units is modified by a shock lying 30 astronomical units beyond (that is roughly at an optical thickness of 3).

In Figure 7 we show the upwind antisolar intensities normalized to the value at 15 astronomical units and computed for the four density distributions of Table 3 . The effect of the existence and the position of the shock is quite clear, showing that in presence of a shock the intensity falls down less quickly than what is expected from a classical hot model.

It can be noted that the radial gradient given for Voyager $2(\gamma=-0.78)$ is obtained for a shock at 54 astronomical units (i.e. the density is multiplied by 2 at 54 astronomical units). As mentioned above, this result is slightly sensitive to the interval of study, though this effect is more important 


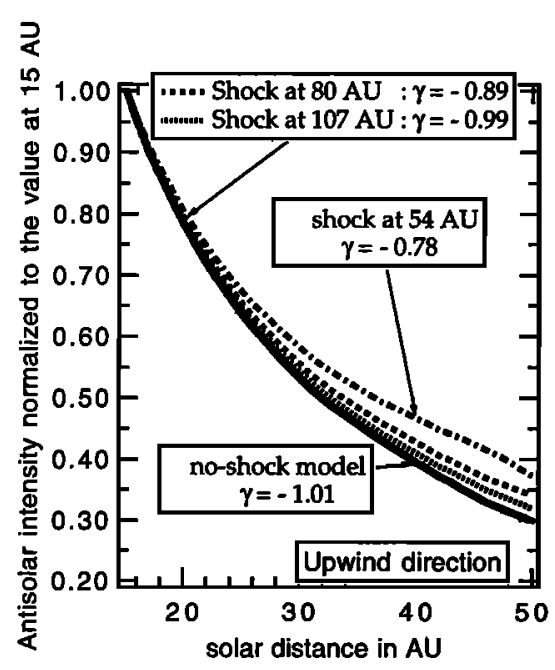

Fig. 7. Antisolar intensities in the upwind direction computed for the four density distributions of Table 3. The curves are normalized to the value at $15 \mathrm{AU}$. We see clearly the effect of the position of the shock on the gradient, namely that a close shock causes the intensity to fall off less quickly than a farther one. $\gamma$ values were computed for solar distances between 15 and $50 \mathrm{AU}$.

downwind. For instance, for the shock at $54 \mathrm{AU}$, we have $\gamma(15-40)=-0.76$ and $\gamma(15-30)=-0.70$. Besides, this result depends also on the fact that we have chosen to multiply the density by a jump factor of 2 . For instance, a jump factor of 1.5 at $\mathbf{5 4}$ astronomical units, with multiple scattering, gives $\gamma=-0.82$. Then, we can expect that a larger jump but more remote than 54 astronomical units will yield the same value as a shock at 54 astronomical units with a 'jump' factor of 2 . As we can see, a more constrained interface effect is necessary to get a precise estimate of the possible interface.

\subsection{Density Distribution Model With a Slow Positive Gradient of Density}

Another example of this uncertainty is whether or not the density jump is superposed with a radial gradient of density in the outer heliosphere. Such a gradient is expected to be positive, and in fact the depletion in interstellar neutral $\mathbf{H}$ entering the heliosphere might be well represented by this gradient alone, without the addition of a jump of density.

To study this possibility, we assumed that in the upwind direction the density number $n(\vec{r})$ between 15 and 100 astronomical units was proportional to $\sqrt{r}$. This assumption was superposed to a hot model with $\mu=0.99$ and $T_{d}=1.210^{6}$ $s$ outside a cone with a half angle of $40^{\circ}$ around the upwind axis. The gradient found between 15 and 50 astronomical units in optically thin approximation was equal to -0.67 and not -0.5 because outside 100 astronomical units the density profile was flat. Using radiative transfer, we found $\gamma=-0.94$, which is in between the values of Table 3 for a shock at 80 astronomical units and a shock at 107 astronomical units. Then, the gradient alone does not enable us to discriminate between a density distribution with a slow positive gradient in the outer heliophere or a shock somewhere near 90 astronomical units. However, the discrepancy between these two density distributions appears when comparisons of intensities measured for different direction of sight at the same point in the heliosphere are considered, as shown in the next section.

\section{InTENSity Maps IN THE OUTER Heliosphene}

In order to derive correctly the $\gamma$ value from a given data set of radial intensities, one must take into account with a high degree of accuracy the possible instrumental calibration drift over long periods of time and the temporal variation of the illuminating solar Lyman $\alpha$ flux at the center of the line. One way to avoid such problems is to study from one point in the heliosphere a full sky Lyman $\alpha$ intensity pattern. Of course, such analysis will depend on the exact position of the spacecraft. Some results of this kind of analysis on the Voyager data have been presented by Lallement et al. [1989].

Here we will study the effect of the wall-type density distribution of Figure 6 on the intensity maps measured for two positions (30 astronomical units and 45 astronomical units in the upwind direction).

In Figure 8 we show the computed intensity map at 30 astronomical units from the Sun, upwind. This computation includes radiative transfer. At variance with maps obtained from the inner heliosphere, the wide maximum region is near the sun, toward the downwind direction, because the maximum emissivity region (MER) is located between the Sun and the spacecraft, at a solar distance of the order of 1 astronomical unit. The upwind region shows a relatively flat minimum of intensity, where we see no conspicuous increase due to the existence of the truncated cone (with an half angle of $40^{\circ}$ ) with double density. Its shape is blurred by multiple scattering and extinction effects. Even computed for a point at a solar distance of 45 astronomical units (Figure 9), the cone feature does not appear clearly.

On the other hand, the presence of the shock modifies strongly the upwind to downwind ratio. Here downwind lines of sight cross the MER, then for a given position of observation $I_{d w}$ will not be sensitive to the existence of a shock in the upwind region. We can then evaluate the solar parameters by studying the downwind hemisphere which is mainly unaffected and then try and see if there is any discrepancy between the upwind intensity pattern and the upwind pattern predicted with a no interface model fitting the downwind side.

In Figure 10 we show the intensities computed at 30 astronomical units from the Sun in the case of a shock at 54 astronomical units from the Sun divided by the intensities computed in the same conditions but in absence of a shock. Because of radiative transfer, the increase of intensity is spread over the sky but in this particular case, its maximum value is of $17 \%$ in the upwind direction (Optically thin computations give a maximum of $60 \%$ in this case). This computation was done also at 45 astronomical units from the Sun, showing a similar pattern though in this case the shock becomes much more apparent. The maximum increase of intensity due to the shock is of $33 \%$ in this case.

So as we have seen here, we have another interesting way to try and determine the presence of an imprint of the heliopause on the neutral hydrogen distributions. Since these maps are made from one point in a short time span, we avoid most of the difficulties of calibration or time variation of the illuminating Lyman $\alpha$ flux. Better constraints on the shape and location of the shock can be obtained by using several of these maps obtained at different points of the spacecraft on its trajectory. 
$\mathrm{Td}=12 \mathrm{E}+07 \mathrm{~s}$

$\mathrm{MU}=0.99$

model : M.S.

shock at $54 \mathrm{AU}$
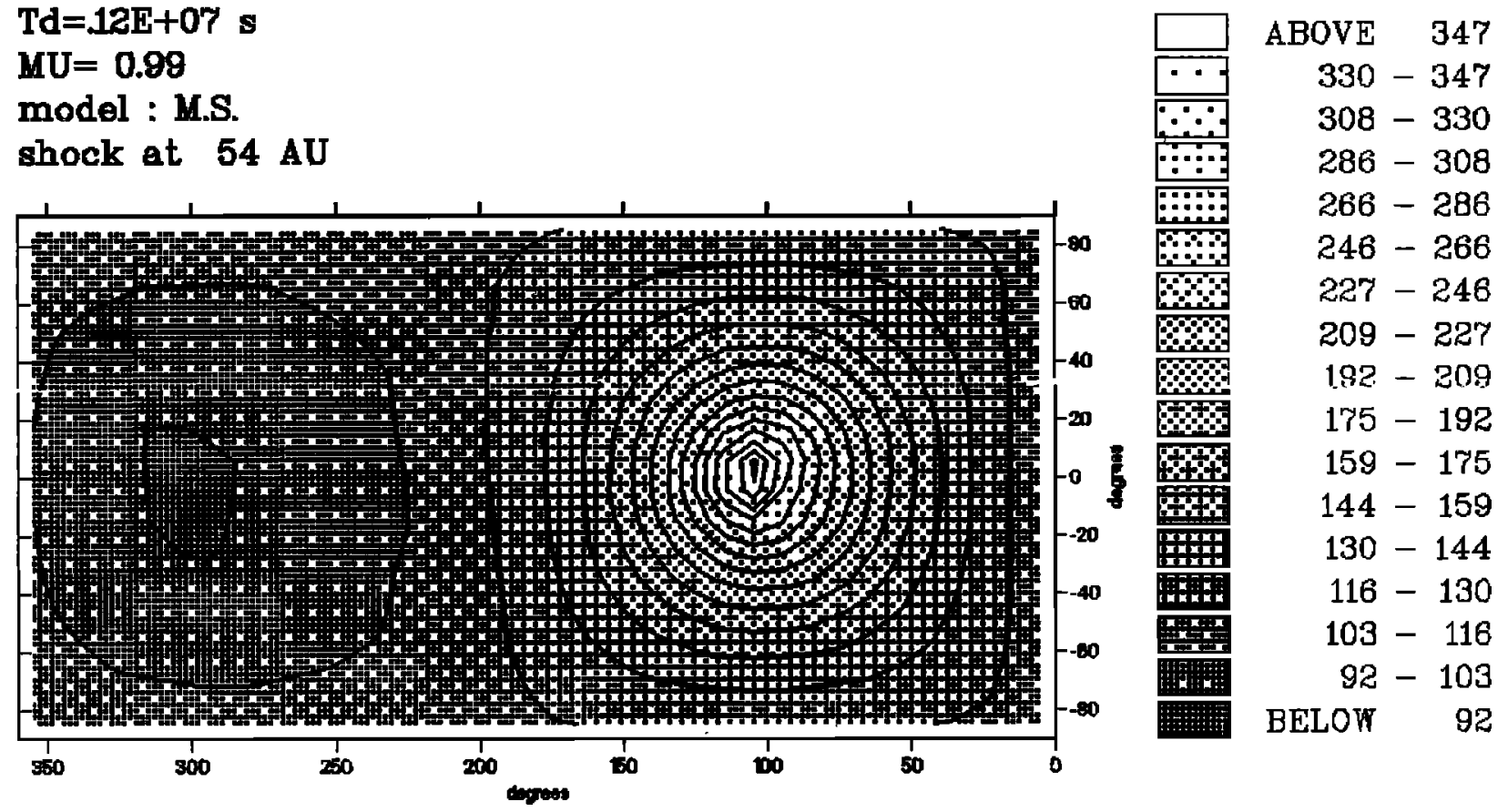

Fig. 8. Isophote map computed at a solar distance of 30 astronomical units close to the upwind direction $(\beta=$ $280^{\circ}$ and $\lambda=0.66^{\circ}$, close to an actual past position of one of the Voyager spacecraft). The maximum emissivity comes from the MER near the sun. The $H$ wall at a solar distance of 54 astronomical units in the upwind direction $\left(\beta=254^{\circ}\right.$ and $\left.\lambda=6^{\circ}\right)$ is not clearly apparent. Units are in Rayleigh.

\section{Td=.12E+or $\mathrm{s}$ \\ $\mathrm{MU}=0.89$ \\ model : M.S. shock at 54 AU}

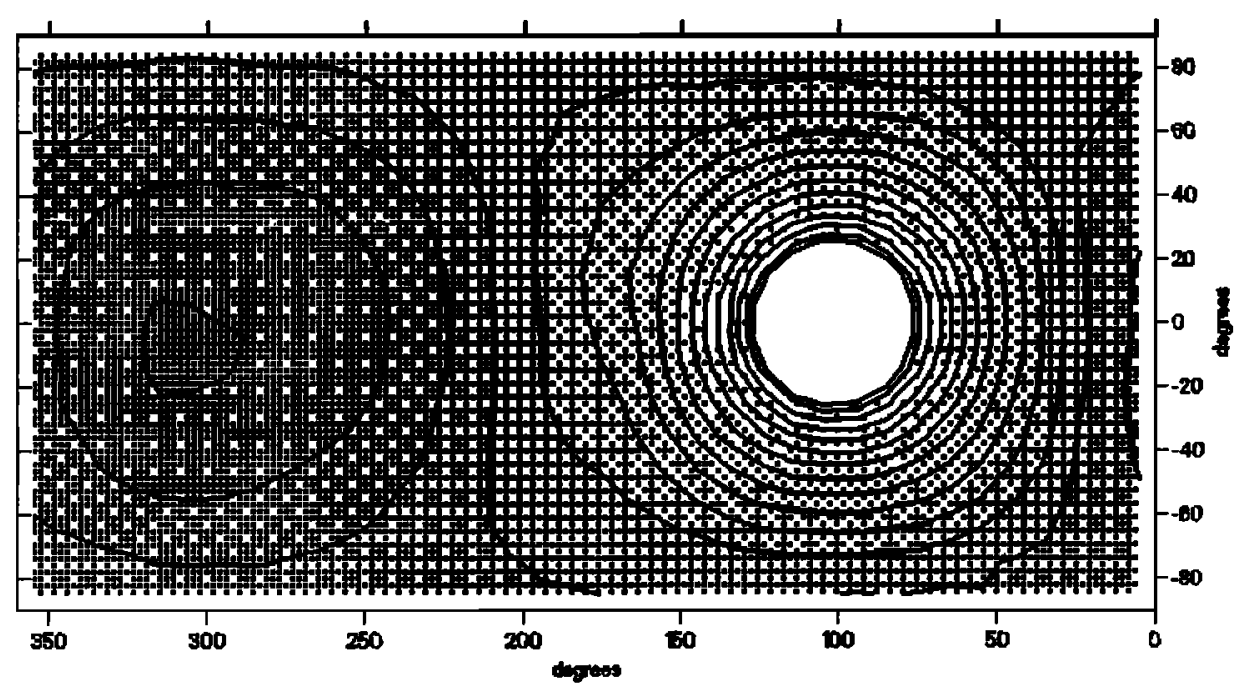

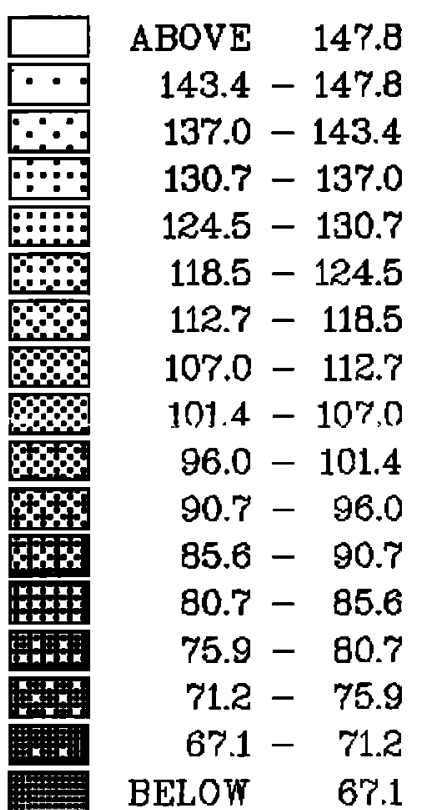

Fig. 9. Same as previous figure. This plot was made from a position of 45 astronomical units (same direction). Units are in Rayleigh. All intensity values outside the linits of 65 and $150 \mathrm{R}$ are not represented here.

\section{Conclusions}

In the early 1970s, the discovery of the $H$ neutral population inside the solar system from Lyman $\alpha$ mapping and its close connection to the interstellar wind yielded no clue of anything special happening near the heliopause, but at least the observed incoming direction told us in which direction to search for the nearest point of the heliopause: upwind.

The $\mathrm{H}$ velocity distribution in the inner solar system, which bears the imprint of the heliopause crossing, can be analyzed with high-resolution measurements of the Lyman $\alpha$ line profile.

The analysis of the interplanetary Lyman $\alpha$ line profiles is a source of information on the heliospheric boundary in two different ways. The first direct way is the measurement of the $H$ velocity vector $\vec{V}_{H}$ by the Doppler shift of the lines. Since the initial interstellar velocity is now rather well es- 

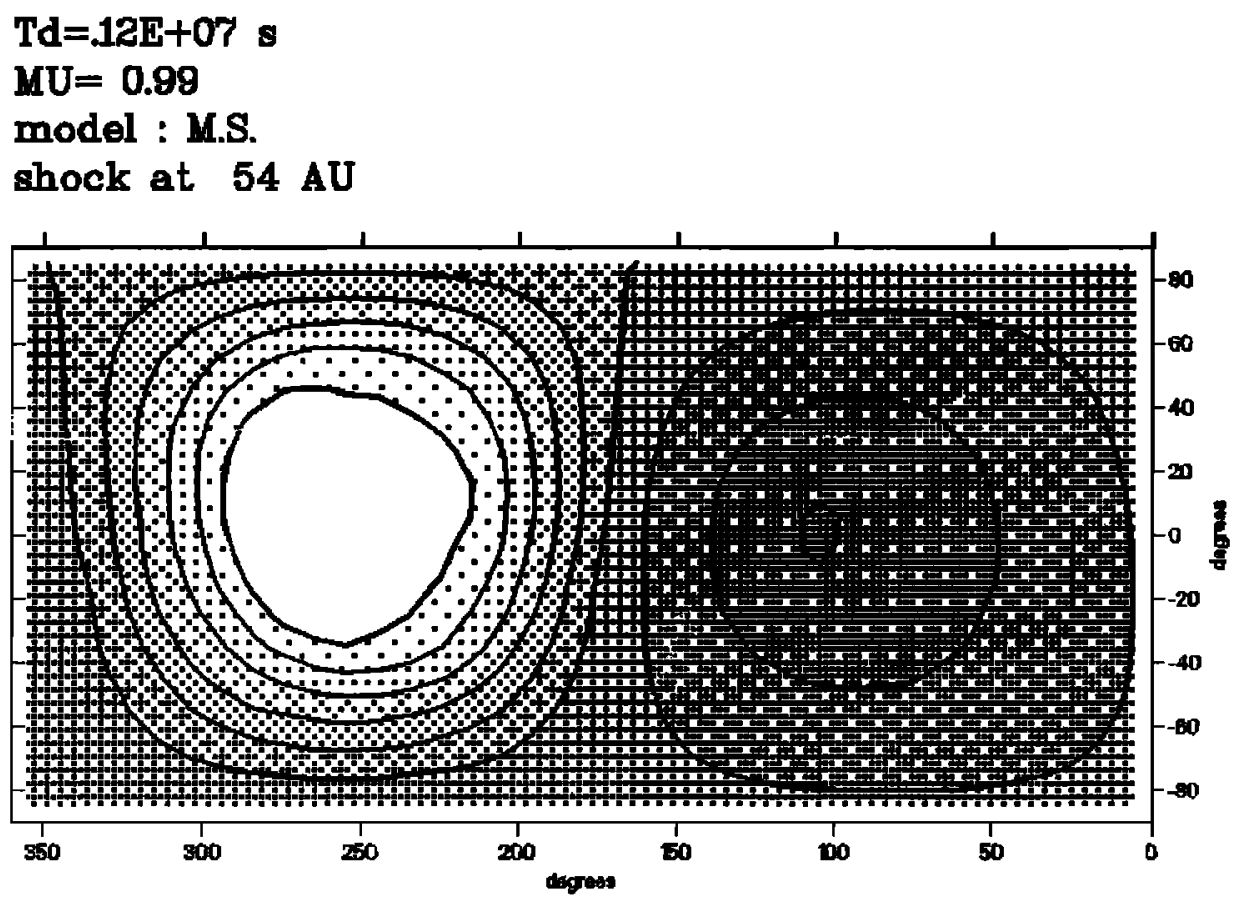



Fig. 10. Isocontours of the ratio of the intensities computed from 30 astronomical units with a radiative transfer model for two different density models. The first model is the $\mathrm{H}$ wall model of Figure $7\left(R_{s}=54 \mathrm{AU}, n_{\infty}=0.1\right.$ $\mathrm{cm}^{-3}$ ). The second one is the classical hot model (i.e., no interface) with the same parameter values as those of the wall model outside the cone of double density. The upwind ratio, in the direction of the $H$ wall, shows a maximum excess of $17 \%$, whereas lines of sight in the opposite direction show almost no excess at all. It must be noted that in this particular case the distance between the wall and the observer is of 24 astronomical units and that the density is doubled at the position of the wall.

tablished to be $V_{\mathrm{LIC}}=26 \mathrm{~km} / \mathrm{s}$ [Lallement and Bertin, 1992; Bertin et al., this issue], it gives an immediate measurement of the $\mathrm{H}$ bulk deceleration $\vec{V}_{\mathrm{LIC}}-\vec{V}_{\mathrm{H}}$ due to charge exchange coupling with protons at the interface. It is then crucial, from this point of view, to confirm earlier hydrogen bulk flow measurements at $20 \mathrm{~km} / \mathrm{s}$ on the upwind side [Bertaux et al., 1985], which imply a rather strong deceleration of 6 $\mathrm{km} / \mathrm{s}$.

On the other hand, the Lyman $\alpha$ profiles analysis probes the heliospheric boundary by itself, just from the directional pattern of the observed $\mathrm{H}$ velocity field. This is true whatever is the distance to the heliopause and heliospheric shock, thanks to the so-called umbrella effect. It certainly deserves accurate measurements of the profiles in all directions.

Hopefully, the analysis of the future SOHO/SWAN H cell results, and maybe of Hubble GHRS spectra, if the lowwavelength side 1 part is repaired, will bring the necessary observations. From the theoretical point of view, this requires to improve the modeling of the $\mathrm{H}$ flow, in particular by including full radiative transfer line profiles calculations. Maybe the comparison between the data and refined plasma interface models should then allow to favor one of them.

On the other hand, if there is a density decrease linked to the heliopause interaction along the wind direction (which is likely, since $\mathrm{H}$ is observed to be decelerated), the Voyager spacecraft will encounter sometime an increase of $\mathrm{H}$ neutral density. This increase may be detected, by Lyman $\alpha$ intensity observations from Voyager UVS, 10 to $\approx 30$ astronomical units before the actual increase, according to the actual extent of this increase, and probably before the solar wind shock is experienced by in situ solar wind instruments.
We have examined two possibilities of diagnostic, both of which requiring the use of radiative transfer modeling. The radial intensity gradient method requires a high stability in the instrumental calibration over long periods of time, and a high degree of confidence in the knowledge of illuminating Lyman $\alpha$ solar line center. Taken at face value, the intensity gradient $\gamma=-0.78$, reported recently by Gangopadhyay and Judge [1992] between 15 and 40 astronomical units, could be explained by a doubling of the density at 54 astronomical units, in which case Voyager would be at the present time very near the inner solar wind shock.

New recording of maps with UVS Voyager, or more simply the distribution of intensity from upwind to downwind (which does not require a roll maneuver, but only scan platform activities) could support such a finding, withtout having to rely on the ratio of measurements taken over a span of nearly 10 years.

Acknowledgmenis. The Editor thanks two referees for their assistance in evaluating this paper.

\section{REFERENCES}

Adams, T. F., and P. C. Frisch, High resolution observations of the Lyman alpha sky background, Astrophys. J., 212,300 , 1977.

Anderson, D. E., and C. W. Hord, Multidimensional radiative transfer; applications to planetary coronae, Planet. Space $S c i$, $25,563,1977$.

Baranov, V. B., Interaction of the solar wind with the external plasma, in Physics of the Outer Heliosphere, edited by S. Grzedzielski and D. E. Page, p. 287, Pergamon, New York, 1990. 
Baranov, V. B., M. G. Lebedev, and J. G. Malama, The influence of the interface between the heliosphere and the local interstellar medium on the penetration of the $H$ atoms to the solar system, Astrophys. J., 375, 347, 1991.

Bertaux, J. L., and J. E. Blamont, Evidence for an extraterrestrial Lyman-alpha emission, the interstellar wind, Astron. Astrophys., 11, 200, 1971.

Bertaux, J. L., R. Lallement, V. G. Kurt, and E. N. Mironova, Characteristics of the local interstellar hydrogen determined from Prognoz 5 and 6 interplanetary Lyman- $\alpha$ line profiles measurements with a hydrogen absorption cell, A stron. Astrophys. $150,82,1985$.

Bertin, P., R. Lallement, R. Ferlet, and A. Vidal-Madjar, Detection of the local interstellar cloud from high-resolution spectroscopy of nearby stars: Inferences on the heliospheric interface, J. Geophys. Res., this issue.

Blum, P. W., and H. J. Fahr, Interaction between interstellar hydrogen and the solar wind, Astron. Astrophys., 4, 280, 1970.

Brandt, J. C., and J. W. Chamberlain, Interplanetary gas, I, hydrogen radiation in the sky, Astrophys. J., 130, 670, 1959.

Chassefière, E., J. L. Bertaux, R. Lallement, and V. G. Kurt, Atomic hydrogen and helium densities of the interstellar medium measured in the vicinity of the sun, Astron. Astrophys. 160, 229, 1986.

Chassefière, E., F. Dalaudier, and J. L. Bertaux, Estimate of interstellar helium parameters from Prognoz 6 and Voyager 1/2. EUV resonance glow measurements taking into account a possible redshift in the solar line profile, Astron. Astrophys., 174, 239, 1988.

Clarke, J. T., S. Bowyer, H. J. Fahr, and G. Lay, IUE high resolution spectrophotometry of $\mathrm{H}$ Lyman alpha emission from the local interstellar medium, Astron. Astrophys., 199, 389, 1984.

Dalaudier, F., J. L. Bertaux, V. G. Kurt, and E. N. Mironova, Characteristics of interstellar helium observed with Prognoz 6 $58.4 \mathrm{~nm}$ photometers, Astron. Astrophys., 134, 184, 1984.

Fahr, H. J., and H. W. Ripken, The physics of the heliospheric interface and its implications for LISM diagnostics, Astron. Astrophys., 139, 551, 1984.

Fahr, H. J., Filtration of the interstellar neutrals at the heliospheric interface and their coupling to the solar wind, in Physics of the Outer Heliosphere, edited by S. Grzedzielski and D. E. Page, p. 327, Pergamon, New York, 1990.

Gangopadhyay, P., and D. L. Judge, The backscattered Lyman-a glow emission as a signature of the termination shock, in Book of Abstracts, World space congress, Washington DC, Committee on Space Programs and Research, Sept. 1992.

Holzer, T.E., Interaction between the solar wind and the interstellar medium, Ann. Rev. Astron. Astrophys., 27, 199, 1989.

Hall, D.T., D. E. Shemansky, D. L. Judge, P. Gangopadhyay, and M. A. Gruntman, Heliospheric hydrogen beyond 15 AU: Evidence for a termination shock, J. Geophys. Res., this issue.

Lallement, R., J. L. Bertaux, and F. Dalaudier, Interplanetary Lyman-alpha spectral profiles and intensities for both repulsive and attractive solar force fields: predicted absorption pattern by a hydrogen cell, Astron. Astrophys., 150, 21, 1985.

Lallement, R., A. Vidal-Madjar A., and R. Ferlet, Multicomponent velocity structure of the local interstellar medium, Astron. Astrophys., 168, 225, 1986.

Lallement, R., and J. L. Bertaux, Deceleration of interstellar hydrogen at heliopause crossing suggested by Lyman- $\alpha$ spectral observations, Astron. Astrophys., 291, L3, 1990.

Lallement, R., R. Ferlet, A. Vidal-Madjar, and C. Gry, Velocity structure of the local interstellar medium, in Physics of the Outer Heliosphere, edited by S. Grzedzielski and D. E. Page, p. 37, Pergamon, New York, 1990.

Lallement, R., J. Malama, E. Quémerais, J. L. Bertaux, and N. A. Zaitzev, Interplanetary Lyman- $\alpha$ spectra as a diagnostic of the local interstellar plasma density: the two-shocks Baranov heliosphere case, Astrophys. J., 396, 696, 1992.

Lallement, R., and P. Bertin, Northern hemisphere observations of nearby interstellar gas: possible detection of the local cloud, Astron. Astrophys., 266, 479, 1992.

Linsky, J. L., A. Brown, K. Gayley, A. Diplas, B. D. Savage, T. R. Ayres, W. Landsman, S. N. Shore, and S. R. Heap, GHRS observations of the local interstellar medium and the deuterium/hydrogen ratio along the line of sight toward capella, Astrophys. $J$., in press, 1993.

Malama, J. G., Monte Carlo simulations of neutral $\mathrm{H}$ atoms in the heliosphere, Astr. Space Science, 176, 21, 1991.

Osterbart, R., and H. J. Fahr, A Boltzmann-kinetic approach to describe the entrance of neutral interstellar hydrogen into the heliosphere, Astron. Astrophys., 264, 260, 1992.

Quémerais, E., R. Lallement, and J. L. Bertaux, Heliospheric modifications of interstellar neutral hydrogen compatible with Lyman- $\alpha$ glow observations, Astron. Astrophys., 265, 806, 1992.

Quémerais, E., and J. L. Bertaux, Radiative transfer at Lyman $\alpha$ in the interplanetary medium: comparison of numerical and Monte Carlo simulations, in Book of Abstracts, World space congress, Washington DC, Committee on Space Programs and Research, Sept. 1992.

Thomas, G. E., Lyman alpha scattering in the Earth's hydrogen geocorona, J. Geophys. Res., 68, 2639, 1963.

Thomas, G. E., and R. F. Krassa, OGO-5 measurements of the Lyman alpha sky background, Astron. Astrophys., 11, 218, 1971.

Thomas, G. E., The interstellar wind and its influence on the interplanetary environment, Ann. Rev. Earth Planet. Sci., 6, $173,1978$.

Wallis, M. K., 1975, Local interstellar medium, Nature, 254, 202, 1975.

Witte, M., H. Rosenbauer, M. Banaszkiewicz, and H. J. Fahr, The Ulysses neutral gas experiment; determination of the velocity and temperature of the interstellar neutral helium, in Book of Abstracts, World space congress, Washington DC, Committee on Space Programs and Research, Sept. 1992.

Wu, F. M., and D. L. Judge, A reanalysis of the observed interplanetary hydrogen Lyman- $\alpha$ emission profiles and the derived local interstellar gas temperature and velocity, Astrophys. J., $299,389,1980$.

J.L. Bertaux, R. Lallement, and E. Quémerais, Service d'aéronomie du CNRS, BP 3, 91371, Verrières le Buisson, France.

(Received October 5, 1992;

revised January 7,1993 ;

accepted February 14, 1993.) 